\title{
Post Processing Hyper-Spectral Data and Generating More Information from X-ray Maps
}

\author{
Richard Wuhrer ${ }^{1}$ and Ken Moran ${ }^{2}$
}

1 University of Western Sydney, Advanced Materials Characterisation Facility (AMCF), Australia 2 Moran Scientific Pty Ltd, 4850 Oallen Ford Road, Bungonia, NSW, 2580, Australia

X-ray mapping (XRM), in the past, was a slow process often being performed over night. With the development of silicon drift detectors (SDD), it is possible to obtain live x-ray images. Currently there are very large area SDD's as well as multiple SDD detector systems capable of handling millions of counts per second. This has made XRM a more popular technique for characterizing a material's microstructural properties by determining elemental distributions and phases in materials with subsequent chemical phase mapping (CPM) [1].

SDD's now allow the electron microprobes to operate at higher beam currents, which are more suited to wavelength dispersive spectroscopy (WDS). Higher spatial resolution mapping is now possible due to larger detection areas, improvements in the field emission guns (FEG) and lower keV mapping.

High pixel resolution x-ray maps of $512 \times 512$ pixels, with excellent counting statistics, can be acquired in a couple of hours. Simple elemental x-ray maps can be misleading, so further post processing (data manipulation) techniques may be required to obtain more information about a material's unique properties.

Our research aims at developing post processing techniques to improve the quantitation of x-ray map data and to develop further post processing techniques for improved characterisation as an aid in assessing the practical properties of complex materials. These techniques include:

1. ZAF corrected quantitative X-ray maps (QXRM) where the QXRM (Fig. 1) enables reliable quantitative results, that when grouped, can be an order of magnitude better than traditional analysis [2].

2. Scatter diagrams and rotational scatter diagrams [2-5] for locating phases.

3. Pseudo colouring (Fig. 1e) for locating phases. The colours of the elements can be rotated, which can often reveal further information (and other phases) in the material. Indeed, the rotating of colours between elements can also show other features, such as hairline cracks, fine precipitates and small boundary interfaces that would be otherwise missed.

4. Filtering to make phase selection easier, to produce cleaner images and aid in trace element location.

5. Theoretical BSE maps. When generating quantitative x-ray maps, the computer can easily calculate the theoretical BSE coefficient for each point on the map. The comparison between the real and theoretical BSE map (image) gives us information about what is going on just below the surface of the sample. We can also ratio the real BSE to the generated BSE to pick up problems such as quantification errors, missing elements, interaction problems and ZAF correction problems.

6. Elemental ratio maps. This is a powerful technique that helps to reveal subtle chemical variations in a sample (Fig. 1c\&d). Ratio maps can also be created from different combinations of elements and from the different background regions near elements of the spectra. 
7. Atomic number-absorption and fluorescence (ZAF) maps. These maps give information on the correction that is required at specific points, or interfaces, of the different regions in the maps.

8. Totals mapping. The totals at each point of the map can be calculated and mapped. These total maps give an idea of the quality of the analysis and also make it easy to determine problems such as missing elements and interaction volume effects.

9. X-ray map resolution determination through the use of Smart J package [6-7], can be used to do x-ray map resolutions for various elements compared to Monte Carlo [8] simulations.

This paper will discuss all of the above mentioned techniques.

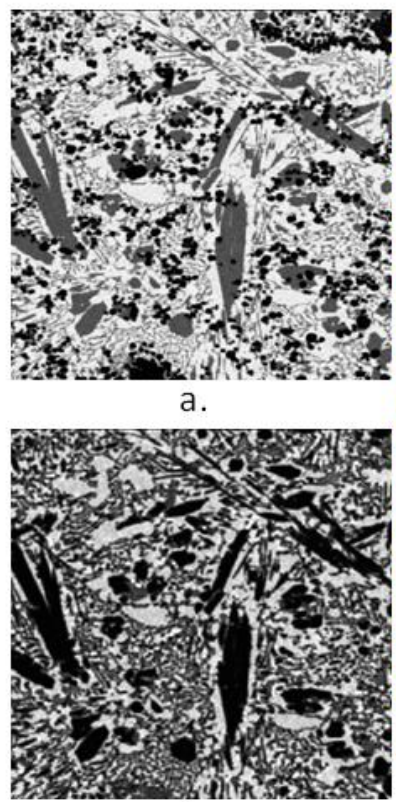

C.

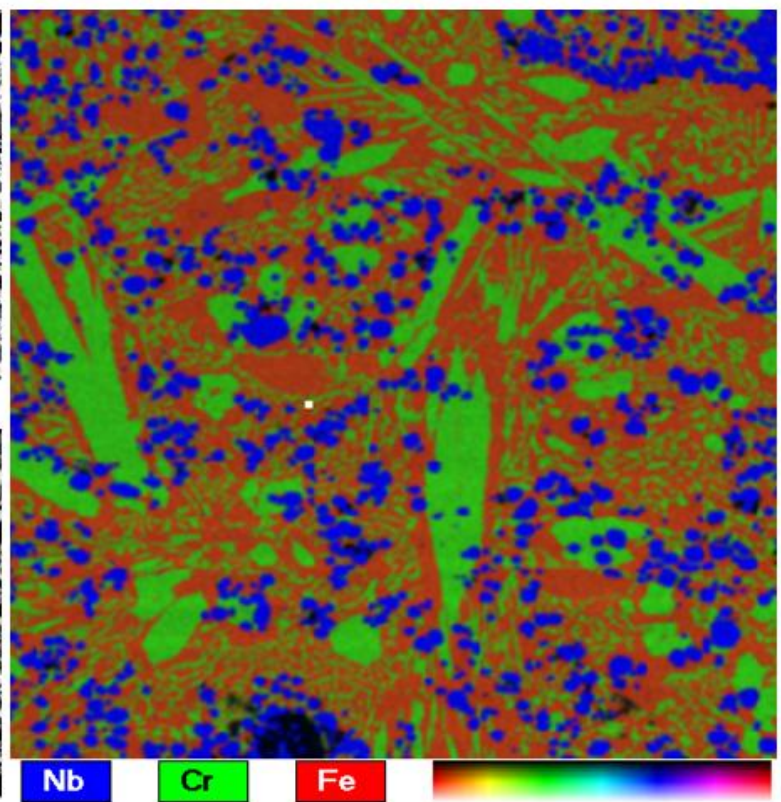

e.

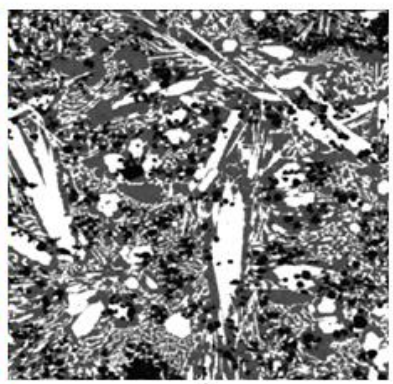

b.

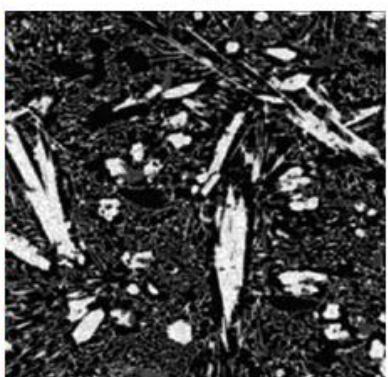

d.

Fig. 1. Hard facing material of $\mathrm{NbC}$ particles in white cast iron. a) Quantitative Iron x-ray map, b) quantitative Chromium x-ray map, c) ratio map of iron to Chromium $(\mathrm{Fe} / \mathrm{Cr}$ ), d) ratio map of Chromium to Iron $(\mathrm{Cr} / \mathrm{Fe})$ showing changes around the eutectic carbides and e) pseudo coloured $\mathrm{x}$ ray map.

\section{References}

[1] R. Wuhrer and K. Moran, "Representation of Microstructures through Rapid X-Ray Mapping", Materials Australia, Volume 38, No 5, September-October (2006) 12-14.

[2] K. Moran and R. Wuhrer, "Quantitative Bulk and Trace Element X-Ray Mapping Using Multiple Detectors", Mikrochimica Acta, Vol. 155, pp. 59-66 (2006).

[3] K. Moran and R. Wuhrer, "X-ray Mapping and Interpretation of Scatter Diagrams", Mikrochimica Vol. 155, pp. 209-217 (2006).

[4] R. Wuhrer, K. Moran and M. R. Phillips, "Multi-Detector X-Ray Mapping and Generation of Correction Factor Images for Problem Solving“, Microscopy and Microanalysis, 14(suppl 2), 1108CD-1109CD (2008).

[5] R. Wuhrer, K. Moran and M. R. Phillips,"X-Ray Mapping and Post Processing“, Microscopy and Microanalysis, 12(suppl 2), 1404CD-1405CD (2006).

[6] IMAGE Java written by Wayne Rasband is available from http://rsb.ifo.nih.gov/ij/ The SMART-J plug-in is available from David Joy (djoy@utk.edu).

[7] D. C. Joy, J. Michael and B. Griffin, "Evaluating SEM performance from the contrast transfer function", Proc. SPIE 7638, Metrology, Inspection, and Process Control for Microlithography XXIV, 76383J (April 15, 2010), doi:10.1117/12.846455.

[8] R. Gauvin and P. Michaud, Microscopy and Microanalysis, 15 (2009), p. 488-489. 